\title{
Face Recognition Using Convolution Based Dwt and CLBP Feature Vectors
}

\author{
Narayan T Deshpande ${ }^{1}$ and Dr. S Ravishankar ${ }^{2}$ \\ ${ }^{I}$ Department of Electronics and Communication, BMS College of Engineering, Bangalore, India, \\ ${ }^{2}$ Department of Electronics and Communication, RV College of Engineering, Bangalore, India,
}

\begin{abstract}
The face recognition is an essential criteria to identify a person in recent advanced security systems. In this paper, we propose efficient Face Recognition using Convolution based DWT and CLBP Feature Vectors. Here database images are first applied with DWT then normalized and convolved with the original image to achieve sharpening of images and filtering of high frequency components. The features thus obtained are applied to CLBP which generates more unique features. The same procedure is repeated for test image to obtain unique test features which are compared with database features using Euclidean Distance. The performance parameters are calculated for standard database and it is observed that the proposed method is better in comparison with the existing methods.
\end{abstract}

Keywords: Convolution, CLBP, DWT, Euclidian Distance, Face etc.

\section{I. introduction}

The Biometric feature based recognition [1] is playing a vital role to secure the data in Cloud Computing. The large number of samples required for Big Data poses a challenging task to security. Biometric traits of a person which are constant throughout life can be considered for identification. Nowadays, Different samples of biometrics are fused to obtain more accurate results to satisfy for Big data Samples. The Biometric samples can also be used for linking various government related schemes which helps in disbursing the fund directly without involvement of middle persons. The Biometric based identification also helps in controlling the economy by cashless transactions. The general biometric system has three sections which are enrollment section, test section and matching section. The sensors with different resolutions are used to capture the biometric traits. The database for the enrollment section is created using extracted features of biometric samples. These samples are stored in single column vector to simplify the matching process. The matching section acts as classifiers to compare the features of data base image and test image features to identify a person.

The face recognition is an important biometric trait as it can be acquired without the knowledge of a person and is useful mainly in surveillance applications. The main challenges of face recognition are covariates like illumination, light intensity variations, emotions etc. The need for accurate recognition is required for above mentioned face covariates. The features are extracted based on spatial, frequency and fusion of both spatial and frequency depending on the application. The proposed method involves face recognition by using Convolution, DWT and CLBP. The face database images are convolved with the normalized output of 2D-DWT on face database images. The output of convolution is applied to CLBP to extract the CLBP features. The test image features obtained are compared with database image features using Euclidian distance.

\section{Literature survey}

In this section, the existing techniques of face recognition are discussed. Bicego et al. [2], proposed a system for face recognition based on Hidden Markov Models and wavelet coding. In this approach a sequence of overlapping sub-images are extracted from each face image and the wavelet coefficients for each of them is computed. The whole sequence is then modeled using Hidden Markov Models. Ahmed Shabaan Samra et al. [3], proposed two methods for face recognition The first method combines the wavelet transform (WT) and fast Fourier transform (FFT) for face images of different features, illuminations and small occlusion, while the second method, combine the WT and discrete cosine transform (DCT) for face images of different scales, poses and rotated images $( \pm 20)$. Vinay Kumar and Shreyas [4] proposed Face recognition with variant pose, illumination and expression is a challenging problem. The Gabor wavelets approach is quite perspective and has several advantages such as invariance to homogenous illumination changes, small changes in head pose and robustness against facial hair, glasses. Lim Song Li and Norashikin Yahya [5] proposed a face recognition system combining features extracted using both Gabor wavelets and SVD. For Gabor wavelets, the extracted feature vectors are selected from only 12 out of 40 Gabor wavelets reducing computational time. For SVD, only the first five singular values are selected and its associated right singular vectors are used as the feature vectors. The combination of Gabor wavelets and SVD offers the advantage of increasing the reliability of the face recognition system. Nitin J. Sanket et al., [6] proposed a novel algorithm called Mirrored Fusion to normalize 
the effects of pose variations in facial images. A unique feature extraction technique called Adaptive Filter Wavelet Transform (AFWT) which is a combination of Stationary Wavelet Transform (SWT) along with Wiener filtering and scaling, Discrete Wavelet Transform (DWT) and Discrete Cosine Transform (DCT) is proposed. AFWT results in low contrast images with prominent features which are desirable for enhanced recognition rate of the FR system.Shridhar S. Shanbhag et al., [7] proposed three novel techniques, viz., Spatial Differentiation (SD), Wavelet Transform based Feature Extraction(WTFE), and Twin Pose Testing Scheme (TPTS), to improve the performance of a FR system. SD is used to enhance the facial features. WTFE uses the shift invariance property of SWT which, along with TPTS, neutralizes pose variations. A Binary Particle Swarm Optimization (BPSO) based feature selection algorithm is used to search the feature space for the optimal feature subset. Fatma Zohra Chelali and Amar Djeradi [8] proposed Gabor and Haar wavelet based feature extraction methods for the extraction of features from facial images. Face recognition experiments were carried out by using Artificial Neural Networks like MLP and RBF classifier. Daniel Madan Raja S et al., [9] proposed human face recognition system affected by varying lighting conditions. To overcome the illumination invariant problems, decomposition method is used. At various scales and frequencies the facial features are extracted by multi resolution property of Discrete Wavelet Transform(DWTs). Fusion of match scores depends on low and high frequency which is based on the human face representation to improve the accuracy in varying lighting conditions. Neelamma K. Patil et al., [10] proposed a novel method of face recognition using de-correlation of local features using Discrete Wavelet Transforms (DWT) which improves the recognition accuracy and avoids generalisability problem caused due to subspace discriminant analysis or statistical learning procedure by using a non-statistical procedure which avoids training step for face samples. The method performs well with images with partial occlusion and images with lighting variations as the local patch of the face is divided into several different patches.Fazael Ayatollahi et al.,[11] proposed multimodal face recognition method which extracts features of rigid and semi-rigid regions of the face using Dual-Tree Complex Wavelet Transform (DT-CWT) DT-CWT decomposes range and intensity images into eight sub images and support vector machine (SVM) is used as the classifier. Thamizharasi Ayyavoo et al., [12] proposed face recognition with head rotation and tilt, lighting intensity and angle, facial expression, aging, partial occlusion. Multi scale technique Discrete Wavelet Transform is used for preprocessing. K Means clustering algorithm is used to cluster the pixels in face image obtained from preprocessing step. Binary threshold is applied in the clusters and performance is tested using Fuzzy K Nearest Neighbor classifier.Rasber D. Rashid et al., [13] proposed a feature extraction algorithm based on wavelets and local binary patterns (LBPs). The proposed method decomposes a face image into multiple subbands of frequencies using wavelet transform. LBP histograms based on the traditional 8-neighbour sampling points are extracted from the approximation sub-band and all LBPHs are concatenated into a single feature histogram to effectively represent the face image. Euclidean distance is used to measure the similarity of different feature histograms and the final recognition is performed by the nearest-neighbor classifier.

\section{Proposed model}

In this section, the proposed model of face recognition using convolution of original face image with DWT and CLBP is introduced. The block diagram of proposed model is shown in Fig. 1.

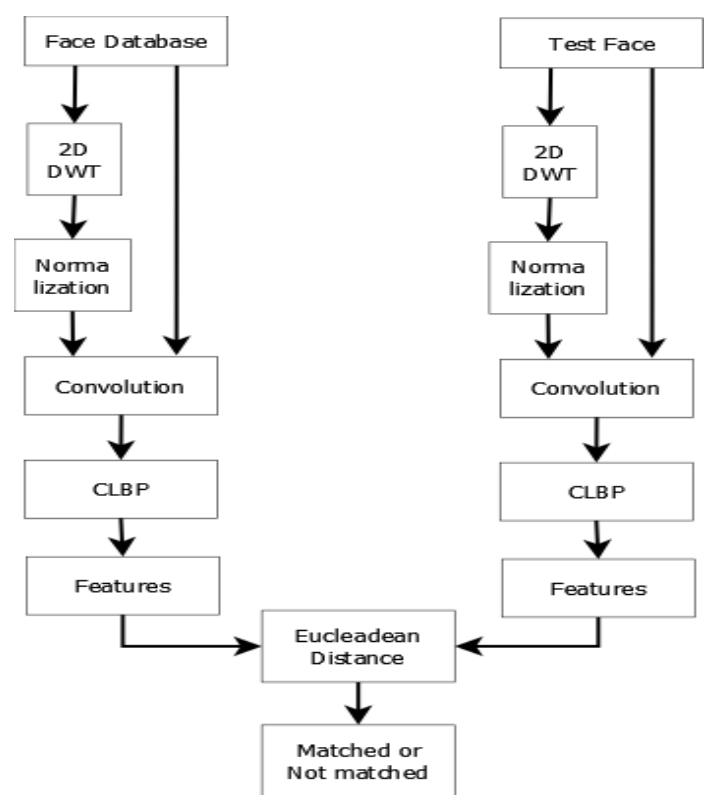

Figure1. Proposed Face Recognition using Convolution and CLBP. 


\subsection{Face Database}

The standard ORL face database is considered to test the proposed model.

\subsection{D-Discrete Wavelet Transform}

The DWT [14] transformation applied on an image will produce compressed image in one of the sub band which is useful in generating reduced number of features. The DWT decomposes an image into four sub bands such as approximation band, vertical band, horizontal band and diagonal band. Firstly, the rows of images are passed through low pass and high pass filter to generate corresponding low and high frequency coefficients. Similarly, the columns of an image are passed through low pass and high pass filter along with rows of low pass filter to generate approximation and vertical bands. The one level DWT decomposition on an image using filter is shown in Fig. 2.

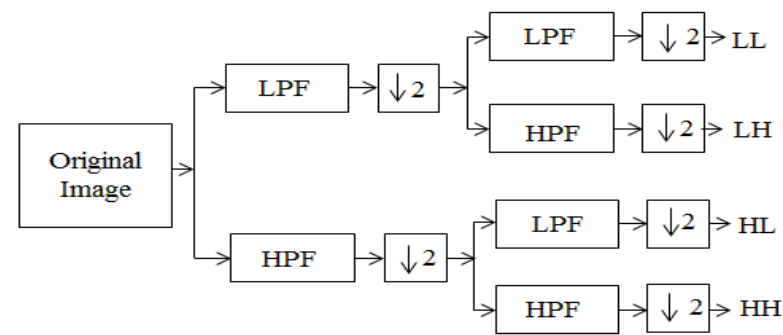

Figure 2. The one level decomposition of DWT using filter

The Haar 2D-DWT of the MxN matrix A is given in equation (1)

$B=W_{M} A W_{N}^{T}$

Let matrix A of size $4 \times 4$ is given in equation (2)

$A=\left[\begin{array}{llll}a_{11} & a_{12} & a_{13} & a_{14} \\ a_{21} & a_{22} & a_{23} & a_{24} \\ a_{31} & a_{32} & a_{33} & a_{34} \\ a_{41} & a_{42} & a_{43} & a_{44}\end{array}\right]$

For partitioning consider $\boldsymbol{W}_{4}=\left[\begin{array}{l}\boldsymbol{H} \\ G\end{array}\right]$ and $\boldsymbol{W}_{4}^{T}=\left[\begin{array}{ll}H^{T} & G^{T}\end{array}\right]$.

Where, $W_{4}=\left[\begin{array}{cccc}\frac{1}{2} & \frac{1}{2} & 0 & 0 \\ 0 & 0 & \frac{1}{2} & \frac{1}{2} \\ -\frac{1}{2} & \frac{1}{2} & 0 & 0 \\ 0 & 0 & -\frac{1}{2} & \frac{1}{2}\end{array}\right]=\left[\begin{array}{l}H \\ G\end{array}\right]$

So, the matrix value of $\mathrm{H}$ and $\mathrm{G}$ is then

$$
\begin{gathered}
H=\left[\begin{array}{cccc}
\frac{1}{2} & \frac{1}{2} & 0 & 0 \\
0 & 0 & \frac{1}{2} & \frac{1}{2}
\end{array}\right] \\
G=\left[\begin{array}{rrrr}
-\frac{1}{2} & \frac{1}{2} & 0 & 0 \\
0 & 0 & -\frac{1}{2} & \frac{1}{2}
\end{array}\right]
\end{gathered}
$$

Then equation (1) becomes $\boldsymbol{B}=\left[\begin{array}{l}\boldsymbol{H} \\ \boldsymbol{G}\end{array}\right] \boldsymbol{A}\left[\begin{array}{ll}\boldsymbol{H}^{\boldsymbol{T}} & \boldsymbol{G}^{\boldsymbol{T}}\end{array}\right]$

$B=\left[\begin{array}{ll}H A H^{T} & H A G^{T} \\ G A H^{T} & G A G^{T}\end{array}\right]$

Where, $\mathrm{HAH}^{\mathrm{T}}=$ Approximation (LL-Band)

$\mathrm{HAG}^{\mathrm{T}}=$ Vertical Difference (LH-Band)

$\mathrm{GAH}^{\mathrm{T}}=$ Horizontal Difference (HL-Band)

$\mathrm{GAG}^{\mathrm{T}}=$ Diagonal Difference $(\mathrm{HH}-\mathrm{Band})$

Substituting the values of equation (1) in equation (3) we get all coefficients values given in equation (4) to equation (7).

$y_{L L}=H A H^{T}=\frac{1}{4}\left[\begin{array}{ll}a_{11}+a_{12}+a_{21}+a_{22} & a_{13}+a_{14}+a_{23}+a_{24} \\ a_{31}+a_{32}+a_{41}+a_{42} & a_{33}+a_{34}+a_{43}+a_{44}\end{array}\right]$
$y_{H L}=H A G^{T}=\frac{1}{4}\left[\begin{array}{ll}\left(a_{12}+a_{22}\right)-\left(a_{11}+a_{21}\right) & \left(a_{14}+a_{24}\right)-\left(a_{13}+a_{23}\right) \\ \left(a_{32}+a_{42}\right)-\left(a_{31}+a_{41}\right) & \left(a_{34}+a_{44}\right)-\left(a_{33}+a_{43}\right)\end{array}\right]$ 


$$
\begin{gathered}
y_{L H}=G A H^{T}=\frac{1}{4}\left[\begin{array}{ll}
\left(a_{21}+a_{22}\right)-\left(a_{12}+a_{11}\right) & \left(a_{23}+a_{24}\right)-\left(a_{13}+a_{14}\right) \\
\left(a_{31}+a_{32}\right)-\left(a_{42}+a_{41}\right) & \left(a_{43}+a_{44}\right)-\left(a_{33}+a_{34}\right)
\end{array}\right] \\
y_{H H}=G A G^{T}=\frac{1}{4}\left[\begin{array}{ll}
\left(a_{11}+a_{22}\right)-\left(a_{12}+a_{21}\right) & \left(a_{13}+a_{24}\right)-\left(a_{23}+a_{14}\right) \\
\left(a_{31}+a_{42}\right)-\left(a_{32}+a_{41}\right) & \left(a_{33}+a_{44}\right)-\left(a_{43}+a_{34}\right)
\end{array}\right]
\end{gathered}
$$

\subsection{Normalization:}

Normalization is used to convert high values of LL band coefficients into lower magnitude values. The normalization is applied on LL sub-band coefficients of an image to convert high coefficients values into lower values. Each LL coefficient values are divided by maximum coefficient value to convert LL coefficient values range from 0 to 1 , which helps in reducing the storage values in real time as given in equation (8).

$$
\text { LL_Normalized coefficient values }=\frac{L L \text { coefficient values }}{\text { maximum value of LL coefficient }}
$$

\subsection{D Convolution:}

The normalized LL sub-band of original face image obtained by DWT is convolved with original face image. The 2D-Convolution is used for sharpening and edge detection of an image effectively. The 2DConvolultion of two images $\mathrm{x}(\mathrm{m}, \mathrm{n})$ and $\mathrm{h}(\mathrm{x}, \mathrm{y})$ is given in equation (9).

$\mathrm{x}(\mathrm{m}, \mathrm{n}) * \mathrm{~h}(\mathrm{~m}, \mathrm{n})=\sum_{i=0}^{w-1} \quad \sum_{j=0}^{H-1} x(i, j) h(m-i, n-j)$

Where $\mathrm{w}$ and $\mathrm{H}$ are the width and height of an image.

The example of 2D-convolution is explained by considering original matrix of size $4 * 4$ and LL band of original matrix of size $2 * 2$ is as follows:

$$
\text { Original matrix }=\left[\begin{array}{cccc}
126 & 98 & 94 & 100 \\
82 & 77 & 96 & 98 \\
80 & 104 & 146 & 129 \\
79 & 78 & 118 & 134
\end{array}\right]
$$

LL band of original Matrix $=\left[\begin{array}{cc}191.5000 & 214.000 \\ 170.5000 & 263.5000\end{array}\right]$

Normalized matrix $=\left[\begin{array}{cc}0.7268 & 0.8121 \\ 0.6471 & 1\end{array}\right]$

Original matrix $4 * 4$ is convolved with $2 * 2$ Normalized matrix to get $5 \times 5$ output convolution matrix given in equation( 13).

Convolution output matrix $=$

$$
\left[\begin{array}{ccccc}
91.57 & 173.55 & 147.90 & 118.08 & 113.70 \\
141.12 & 311.96 & 291.12 & 333.77 & 219.59 \\
111.19 & 272.37 & 329.68 & 371.73 & 202.76 \\
109.17 & 268.14 & 347.57 & 422.68 & 237.82 \\
51.11 & 129.47 & 154.35 & 204.70 & 134
\end{array}\right]
$$

The linear convolution matrix has unique coefficient values compared to original matrix coefficient values; hence the concept of convolution is used for better classification of images.

\subsection{Compound Linear Binary Pattern (CLBP):}

The CLBP operator [15] is used to obtain the CLBP feature from neighboring pixel values. The CLBP operates with window of odd number size N X N. The center pixel is considered as reference to obtain unique feature by using neighboring pixel values to compute both sign and magnitude components.

Let the centre pixel intensity value be $K c$ and surrounded neighbor pixel intensity values say $K p$. The sign bit patterns for $3 \times 3$ matrixes are generated using Equation 14 .

$S(I)= \begin{cases}0: & K p-K c \leq 0 \\ 1: & K p-K c>0\end{cases}$

(14) 
The magnitude bit pattern is generated using Equation 15.

$M(I)=\left\{\begin{array}{l}0: K p-K c \leq M_{\text {avg }} \\ 1: K p-K c>M_{\text {avg }}\end{array}\right.$

Where $\mathbf{M}_{\text {avg }}=(|\mathrm{m} 1|+|\mathrm{m} 2|$ $.+|\mathrm{m} 8|) / 8$

$\mathrm{m} 1$ to $\mathrm{m} 8$ are the magnitude values of difference between respective $K p$ and $K c$.

The example for CLBP is as shown is Figure 3 which shows unique features for both sign and magnitude component calculated for center pixel value and further this is repeated for all image pixel values in an image.

\begin{tabular}{|c|c|c|}
\hline 9 & 12 & 34 \\
\hline 10 & 25 & 28 \\
\hline 99 & 64 & 56 \\
\hline
\end{tabular}

(a)

\begin{tabular}{|c|c|c|}
\hline-1 & -1 & 1 \\
\hline-1 & & 1 \\
\hline 1 & 1 & 1 \\
\hline
\end{tabular}

(c)

(a) 3x3 sample block

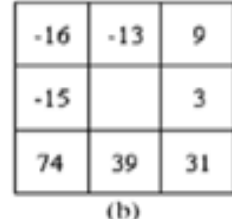

\begin{tabular}{|l|l|l|}
\hline 16 & 13 & 9 \\
\hline 15 & & 3 \\
\hline 74 & 39 & 31 \\
\hline \multicolumn{3}{|c|}{ (d) }
\end{tabular}

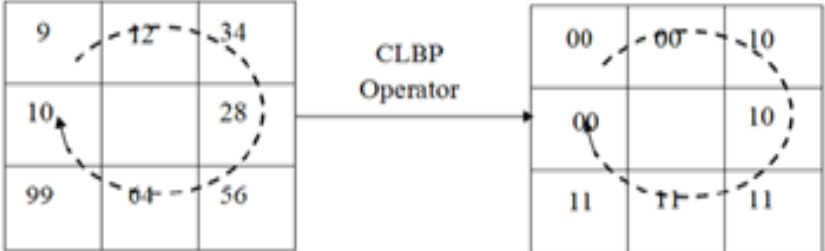

(e)

Fig. 3: CLBP operator

(b) The local difference (c) The sign components (d) Magnitude components (e) original matrix (f) CLBP Matrix

\subsection{Features}

The features are extracted from database face images and test image to form single column feature vector. The single column vector helps in reducing the matching speed using Euclidean distance method.

\subsection{Euclidean distance:}

The distance between database features $\left(\mathrm{p}_{\mathrm{i}}\right)$ and test features $\left(\mathrm{q}_{\mathrm{i}}\right)$ is given in equation $(16)$.

$E D=\sqrt{\sum_{i=1}^{N}\left(p_{i}-q_{i}\right)^{2}}$

Where, $\mathrm{N}=$ No of coefficients in a vector.

$\mathrm{Pi}=$ Coefficient values of vectors in the database.

qi=Coefficient values of vectors in the test image.

The ED is used to find similarities and dissimilarities among face images to test performance of biometric system.

\section{Performance analysis}

In this section, the definitions of performance parameters and experimental results of various techniques are discussed.

\subsection{Definition of Performance Parameters:}

4.1.1 False Rejection Rate (FRR): It is defined as the probability of genuine person being rejected as an imposter and is computed in equation (17).

$$
\mathrm{FRR}=\frac{\text { numberofgenuine }}{\text { person rejected }}
$$

4.1.2 False Acceptance Rate (FAR): Is defined as the probability of imposter being accepted as genuine persons. It is ratio of imposters accepted as genuine persons from outside the database to the total number of persons in the outside database and is computed in equation (18). 
$\mathrm{FAR}=\frac{\text { numberof imposter accepted as genuine person }}{\text { total number of persons in the outside database }}$

4.1.3 Equal Error Rate (ERR): Is define as error tradeoff between FRR and FAR i.e., FAR = FRR for a particular threshold value. A lower EER value indicates better performance of biometric systems.

4.1.4 Total Success Rate (TSR): Is the measure of accuracy of biometric systems. It is the ratio of the total number of genuine persons identified correctly in the data base to the total number of persons inside the database and is computed in equation (19).

$T S R=\frac{\text { genuine persons identified correctly }}{\text { total number of persons inside the database }}$

4.1.5Optimum Total Success Rate (Opt.TSR): The value of TSR corresponding to the EER for specified threshold.

\subsection{Comparison of Proposed Method with existing Methods:}

The percentage TSR of proposed method for ORL database is compared with existing algorithms presented by Pallavi D.Wadakar and MeghaWankhade [16], Swarup Kumar Dandpat and Sukadev Meher[16], Murugan et al.,[18] and Sagar et al.,[19] is given in Table 1. It is observed that the percentage TSR is high in the case of proposed method compared to existing algorithms. The performance of proposed method is better compared to existing algorithms for the following reasons. (i) Normalization performed on 2D DWT scales the coefficient values. ii). Convolution generates unique features. iii). CLBP performed makes the features more unique.

Table 1: Comparison of TSR with proposed and existing algorithms

\begin{tabular}{|l|l|c|l|}
\hline Sr. No & \multicolumn{1}{|c|}{ Authors } & Techniques & \% TSR \\
\hline 1 & Pallavi and Megha [16] & DWT & 90 \\
\hline 2 & Swarup and Sukadev [17] & PCA+2DPCA & 90.5 \\
\hline 3 & Murugan et al.,[18] & Gabor Filter +DWT + PCA & 92 \\
\hline 4 & Sagar et al.,[19] & Convolution+ FCV & 93.33 \\
\hline 5 & Proposed Method & Convolution+ DWT+ CLBP & 94.5 \\
\hline
\end{tabular}

\section{Conclusion}

Face recognition plays a crucial role in many applications which are essential and integral part of life and hence demands a high accuracy in recognizing the desired individual. An efficient approach for face recognition using convolution based DWT and CLBP feature vectors is proposed in this paper. A total success rate of $94.5 \%$ is realized with the proposed method and hence can be considered in comparison with existing methods.

\section{References}

[1]. K. Anil Jain and A. Ross, "Learning User Specific Parameters in a Multi biometric Systems", IEEE Proceedings of International Conference on Image and Signal Processing, pp. 1-5, 2002.

[2]. M. Bicego, U. Castellani, and V. Murino, "Hidden Markov Models and Wavelets for face recognition", 12th International Conference on Image Analysis and Processing (ICIAP), pp. 52-56, 2003.

[3]. Ahmed Shabaan Samra, Salah El Taweel Gad Allah and Rehab Mahmoud Ibrahim, "Face Recognition Using Wavelet Transform, Fast Fourier Transform and Discrete Cosine Transform", IEEE $46^{\text {th }}$ Midwest symposium on circuits and systems, vol. 1, pp. 272$275,2004$.

[4]. Vinay Kumar. B and Shreyas. B. S, "Face Recognition Using Gabor Wavelets", Asilomar conference on signals, systems and computer, pp. 593-597, 2006.

[5]. Lim Song Li and Norashikin Yahya, "Face Recognition Technique using Gabor Wavelets and Singular Value Decomposition (SVD)", IEEE International Conference on Control System, Computing and Engineering, Penang, Malaysia, 2014.

[6]. Nitin J. Sanket, Vyshak A. V, K. Manikantan and S. Ramachandran, "Face Recognition using Adaptive Filter Wavelet Transform based Feature Extraction”, International Conference on Science, Engineering and Management Research”, 2014.

[7]. Shridhar S. Shanbhag, Sourabh Bargi, K. Manikantan and S. Ramachandran, "Face Recognition using Wavelet transforms-based Feature Extraction and Spatial Differentiation-based Pre-processing”, International Conference on Science, Engineering and Management Research, 2014.

[8]. Fatma Zohra and Amar, "Face recognition system using Neural Network with Gabor and discrete wavelet transform parameterization”, IEEE International Conference of Soft Computing and Pattern Recognition, 2014.

[9]. Daniel Madan, Raja S, .Amitabh Wahi, S.Yamuna and L.Priyanga Devi, "Human Face Recognition Under Varying Illumination Condition Using Wavelet Transform", International Conference on Intelligent Computing Applications, 2014.

[10]. Neelamma K. Patil, Vasudha S and Lokesh R. Boregowda, "Performance Improvement of Face Recognition System by Decomposition of Local Features using Discrete Wavelet Transforms", International Symposium on Electronic System Design, 2013.

[11]. Fazael Ayatollahi, Abolghasem A. Raie and Farshid Hajati, "Multimodal Expression-Invariant Face Recognition Using Dual-Tree Complex Wavelet Transform", Iranian Conference on Machine Vision and Image Processing (MVIP ), 2013.

[12]. Thamizharasi Ayyavoo and Jayasudha J.S, "Face Recognition using Enhanced Energy of Discrete Wavelet Transform", 
International Conference on Control Communication and Computing (ICCC), 2013.

[13]. Rasber D. Rashid, Sabah A. Jassim and Harin Sellahewa, "LBP Based On Multi Wavelet Sub-Bands Feature Extraction used for Face Recognition", International Workshop On Machine Learning For Signal Processing, 2013.

[14]. ArvindBisht and Monika Gupta, "Chip Design of DWT for Image Compression", International Research Journal of Engineering and Technology, vol.2, No.4, pp 320-324, 2015.

[15]. Zhenhua Guo, Lei Zhang and David Zhang, "A Completed Modelling of Local Binary Pattern Operator for Texture Classification”, IEEE Transactions on Image Processing, vol. 19, no. 6, pp. 1657-1663, 2010.

[16]. Pallavi D.Wadkar and MeghaWankhade, "Face Recognition using Discrete Wavelet Transforms," International Journal of Advanced Engineering Technology, vol. 3,pp. 239-242, 2012.

[17]. Swarup Kumar Dandapat and SukadevMeher, "Performance Improvement for Face Recognition using PCA and Two-Dimensional PCA, "IEEE International Conference on Computer Communication and Informatics, pp. 1-5, 2013.

[18]. D Murugan, S Arumugam, K Rajalakshmi and Manish T, "Performance Evaluation of Face Recognition using Gabor Filter, Log Gabor filter and Discrete Wavelet Transform, " International Journal of computer science and Information Technology, vol. 2, no. 1, pp. 125-132, 2010.

[19]. Ganapathi V Sagar, Savitha Barker, K B Raja, K Suresh Babu and Venugopal K R, “Convolution based Face Recognition using DWT and Feature Vector Compression”, Third International Conference on Image Information Processing, pp. 444-449, 2015. 